\title{
Towards a Color Prediction Model for Printed Patches
}

Patrick Emmel and Roger David Hersch

Ecole Polytechnique Fédérale de Lausanne (EPFL),

Switzerland

Reprinted from

IEEE Computer Graphics and Applications

Vol. 19, No. 4, July/August 1999

(C) 1999 IEEE. Institute of Electrical and Electronics Engineers.

Personal use of this material is permitted. However,

permission to reprint/republish this material for advertising or promotional purposes or for creating new collective works for resale or redistribution to servers or lists, or to reuse any copyrighted component of this work in other works must be obtained from the IEEE. 


\section{Towards a Color Prediction Model for Printed Patches}

day, calibrating a color printer requires
measuring a minimum of 1,000 patches that sample the whole color space. By processing the collected data, you can generate look-up tables that convert input color data (for instance, calibrated RGB or CIE-XYZ) into the printer's output data (CMYK). Since printing devices exhibit a highly nonlinear behavior, getting reasonable precision requires measuring a large number

\section{A novel color prediction}

\section{model unifies within a}

\section{framework based on matrices}

the phenomena of surface

reflection, light absorption,

diffuse light sources,

\section{superposition of multiple ink}

layers, and others. of samples. Furthermore, the inputoutput transformation of color data depends on many different parameters: the spectra of the inks, the properties of the paper, the halftoning method, and so on. Hence, changing any of these parameters requires performing a new calibration, for example before using a new kind of paper, a new ink cartridge, or a different halftoning algorithm. This may lead to tedious repetitions of the calibration process and make high-quality calibration impractical.

To circumvent this problem, color printer calibration should employ a physical model of the light reflection process, which predicts the color of printed patches. This would allow computing a new calibration profile with only a few measurements. Future generations of printers equipped with sensors that provide the necessary data will then recalibrate themselves automatically.

The literature usually presents the classical models describing the phenomena involved in color prediction separately. This article aims to unify these models into a single framework through an adequate mathematical formulation. We'll successively integrate into the model one phenomenon after the other. The resulting unified physical model should be able to predict the diffuse reflection spectrum of color samples illuminated by a diffuse light source.

In this study, we limit ourselves to predicting uniform color samples made of one or several superposed ink

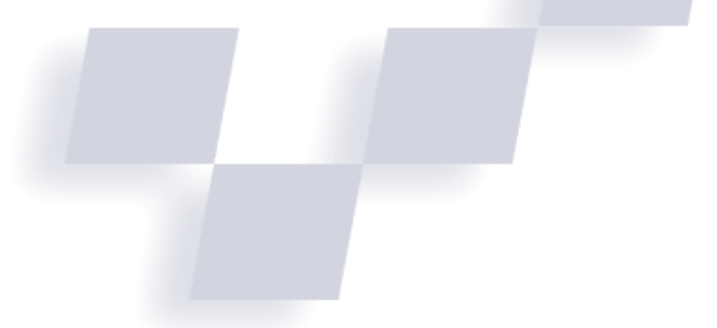

Patrick Emmel and Roger David Hersch Ecole Polytechnique Fédérale de Lausanne (EPFL), Switzerland

layers. We're currently investigating an extension of the proposed model for halftoned samples.

\section{Light absorption}

The most widely known classical model for the absorption of light is the Beer-Lambert-Bouguer law. ${ }^{1}$ This model describes the intensity variation of a light beam crossing a medium that contains identical lightabsorbing particles at a concentration $c$. Let's consider an infinitely thin slice of thickness $d x$ of this medium (see Figure 1). The model relies on the assumption that the particles are independent. According to the BeerLambert-Bouguer law, the variation $d \phi$ of the flux that crosses this slice is proportional to the concentration $c$, to the flux $\phi$ of the beam, and to the thickness $d x$ of the slice. Hence, the flux of a collimated light beam $\phi$ that crosses the infinitely thin layer varies as follows:

$$
d \phi=-\varepsilon(\lambda) \cdot c \cdot \ln (10) \phi d x
$$

where the proportionality coefficient $\varepsilon(\lambda)$ is the molar extinction coefficient of the absorbing particles. Equation 1 is a linear differential equation of the first kind whose solution is given by an exponential function. This kind of function will play a central role in our model. The integration of Equation 1 over a layer of thickness $d$ leads to

$$
\begin{aligned}
\phi(d) & =\exp [-d \cdot c \cdot \varepsilon(\lambda) \cdot \ln 10] \cdot \phi(0) \\
& =10^{-[d \cdot c \cdot \varepsilon(\lambda)]} \cdot \phi(0)
\end{aligned}
$$

The transmission coefficient $T(\lambda)$ is then deduced by dividing the flux $\phi(d)$ by the incoming flux $\phi(0)$ :

$$
\begin{aligned}
T(\lambda) & =\frac{\phi(d)}{\phi(0)}=\exp [-d \cdot c \cdot \varepsilon(\lambda) \cdot \ln 10] \\
& =10^{-[d \cdot c \cdot \varepsilon(\lambda)]}
\end{aligned}
$$

The value $T(\lambda)=1$ corresponds to a transparent medium, whereas the value $T(\lambda)=0$ means no transmission of light and hence an opaque medium. Beer's law is often expressed in its logarithmic form. The absorption (or optical density) is proportional to the path length $d$, 
the concentration $c$, and the extinction coefficient $\varepsilon(\lambda)$. Hence, the optical density $D(\lambda)$-the sign-inverse of the decimal logarithm of the transmission $T(\lambda)$ —equals

$$
D(\lambda)=d \cdot c \cdot \varepsilon(\lambda)
$$

In the density scale, $D(\lambda)=0$ corresponds to a transparent medium, and the values of $D(\lambda)$ increase logarithmically when the transparency decreases. The extreme case of an opaque medium leads to an infinite density $D(\lambda)=\infty$.

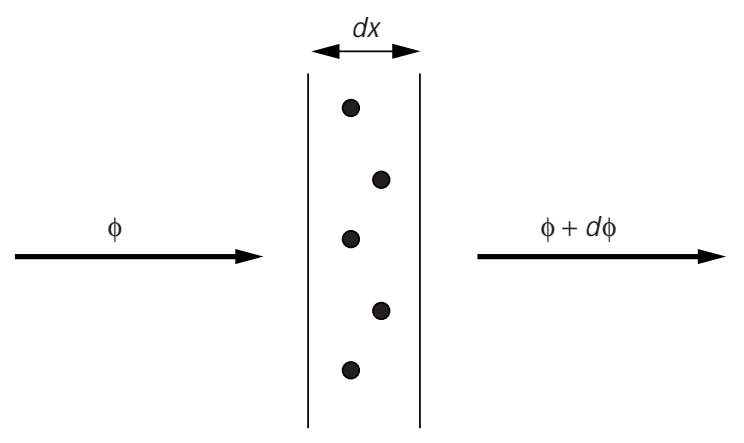

1 Absorption of light by an infinitely thin layer containing light absorbing particles at a concentration c.

\section{Diffuse light and diffuse reflector}

The absorption law presented in the previous section is defined for a collimated light beam - a beam with parallel rays that follow the same path. However, natural light exhibits a rather diffuse behavior, where rays don't have a privileged orientation. A punctual diffuse source or reflector emits rays in all directions of space. A planar source or reflector emits into all directions of one hemisphere. In this section, we analyze the quantitative properties of this last kind of light emitter.

First let's recall the definitions of a few radiometric quantities (see Wyszecki and Stiles, ${ }^{2}$ p. 2). A surface element $d s$ receiving a light flux $d \phi_{r}$ is under an irradiance $E=d \phi_{r} / d s$. A surface element $d s$ of radiance $L$ emits a flux $\phi_{e}$ in a solid angle $d \omega$ making an angle $\theta$ with the normal to the surface:

$$
L=\frac{d^{2} \phi_{e}}{\cos \theta d \omega d s}
$$

Finally, the intensity $I$ of a light flux $\phi$ in a solid angle $d \omega$ is defined by $I=d \phi / d \omega$.

The radiance $L$ of a perfect diffuse reflector doesn't depend on the angle of observation $\theta$; it's related to the irradiance $E$ by the relation $L=E / \pi$ (see Wyszecki and Stiles, ${ }^{2}$ p. 274). Such a reflector is called a Lambert surface (see Wyszecki and Stiles, p. 273). ${ }^{2}$ Therefore we have

$$
\frac{d^{2} \phi_{e}}{\cos \theta d \omega d s}=\frac{E}{\pi}
$$

In the literature (Born and Wolf, ${ }^{3}$ p. 182), this formula-called Lambert's cosine law-is often presented in terms of intensity:

$$
d I=\frac{d^{2} \phi_{e}}{d \omega}=\frac{E}{\pi} \cos \theta \cdot d s
$$

Equation 7 implies that the indicatrix (locus of the extremities of the intensity vectors) of such a surface is a circle (see Figure 2a). We can compute the flux emitted in the direction given by the angle $\theta$ by considering

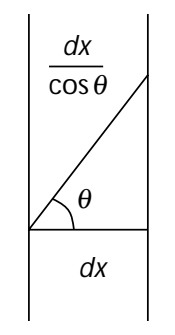

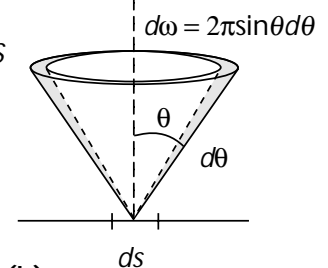

(b)

\begin{abstract}
2 According to Lambert's cosine law, the intensity I of the light emitted by a diffuse reflector $S$ depends only on the cosine of the angle $\theta$ of observation (b). The indicatrix of such a reflector is a circle (a).
\end{abstract}

\section{The average absorption of an infinitely thin slice under diffuse illumination is related to the average path of the light in the medium. The calculation shows that the optical density is twice the density observed for a collimated light beam.}

the solid angle $d \omega=2 \pi \sin \theta d \theta$ (see Figure $2 \mathrm{~b}$ ). A surface $S$ that receives from the upper hemisphere a total flux $\phi_{r}=\int_{S} E d s$ emits a diffuse flux $\phi_{e}$ whose angular distribution is given by ${ }^{4}$

$$
\frac{1}{\phi_{r}} \frac{\partial \phi_{e}}{\partial \theta}=2 \sin \theta \cos \theta=\sin 2 \theta
$$

We'll use this fundamental result for further calculations. Now, we can calculate the average absorption $A$ of an infinitely thin slice of an absorbing medium under diffuse illumination (see Figure 3). From Equation 8 we know the angular distribution of the diffuse light flux. Beer's law gives the absorption in the direction $\theta$, which equals

$$
\frac{d x}{\cos \theta} \cdot \varepsilon(\lambda) \cdot c
$$

Hence we can compute the average absorption $A$ by integrating over all directions:

$$
\begin{aligned}
A & =\int_{0}^{\frac{\pi}{2}}\left(\frac{d x}{\cos \theta} \cdot \varepsilon(\lambda) \cdot c \sin 2 \theta\right) d \theta \\
& =(2 \cdot d x \varepsilon(\lambda) \cdot c) \int_{0}^{\frac{\pi}{2}} \sin \theta d \theta \\
& =2 \cdot d x \cdot \varepsilon(\lambda) \cdot c
\end{aligned}
$$




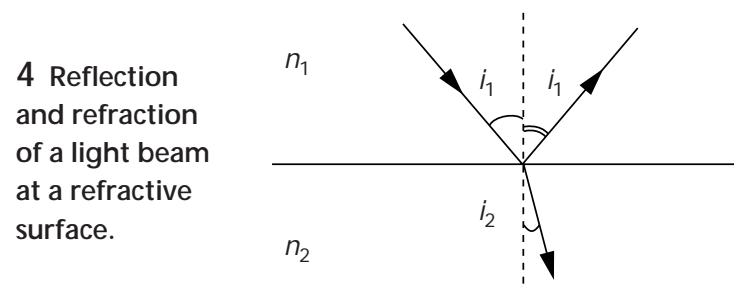

The calculation made in Equation 9 shows that the optical density under diffuse illumination is twice the density observed for a collimated light beam. This fundamental result gives us a generalization of Beer's law for diffuse light:

$$
d \phi=-2 \varepsilon(\lambda) \cdot c \cdot \ln (10) \phi d x
$$

\section{Interface reflection under diffuse light}

Before we study the influence of the interface between media of different refractive index, let's recall the basic laws of light reflection. A light beam that hits a refractive surface with an incidence angle $\theta$ is partially reflected and partially refracted into the other medium (see Figure 4). The reflected beam with the normal to the surface makes the same angle $i_{1}$ as the incident beam, whereas the refracted beam with the normal makes an angle $i_{2}$ related to $i_{1}$ by Snell's law:

$$
n_{1} \sin \left(i_{1}\right)=n_{2} \sin \left(i_{2}\right)
$$

where $n_{1}$ and $n_{2}$ represent the refractive indices of the two media. Note that for $n_{1}>n_{2}$, there exists a limit angle $i_{1 \max }=\operatorname{asin}\left(n_{2} / n_{1}\right)$ over which the incident beam reflects totally.

We can calculate the amplitude of the reflected beam by considering two polarized electromagnetic waves, one polarized parallel and the other perpendicular to the plane of incidence. It can be shown (see Born and Wolf, ${ }^{3}$ p. 40) that the reflection coefficient $r_{a}$ for the parallel polarized wave and the reflection coefficient $r_{e}$ for the perpendicular polarized wave are given by

$$
r_{a}=\left(\frac{\tan \left(i_{1}-i_{2}\right)}{\tan \left(i_{1}+i_{2}\right)}\right)^{2} \text { and } r_{e}=\left(\frac{\sin \left(i_{1}-i_{2}\right)}{\sin \left(i_{1}+i_{2}\right)}\right)^{2}
$$

where $i_{1}$ represents the angle of incidence and $i_{2}$ the angle of refraction according to Snell's law (see Equation 11 and Figure 4). The literature terms these the Fresnel relations. We use $r_{n_{1}, n_{2}}\left(i_{1}\right)$ to denote the reflection coefficient of a beam propagating in a medium of refractive index $n_{1}$, which has an incidence angle $i_{1}$ on the refractive surface delimited by a medium of index $n_{2}$. Since we can consider natural light as an equal mixing of both types of waves, its reflection coefficient equals the mean value of $r_{a}$ and $r_{e}$ :

$$
r_{n_{1}, n_{2}}\left(i_{1}\right)=\frac{r_{a}+r_{e}}{2}
$$

Let's now compute an average reflection coefficient $r_{n_{1}, n_{2}}$ for diffuse light arriving on a plane refractive surface. As we saw earlier, we can calculate such an average by integrating over all directions the angular distribution of diffuse light given by Equation 8 multiplied by the reflection coefficient of a natural light beam given by Equation 13:

$$
r_{n_{1}, n_{2}}=\int_{0}^{\frac{\pi}{2}}\left(r_{n_{1}, n_{2}}(\theta) \cdot \sin 2 \theta\right) d \theta
$$

Judd $^{5}$ did this calculation in 1942 for a large number of refractive indices. A typical result is the particular case of the surface reflection between air and plastic, where the refractive index of air is $n_{1}=1$ and the refractive index of plastic is $n_{2}=1.5$. The computation of Equation 14 leads in this case to $r_{1,1.5}=0.0919$. This value expresses the average reflection coefficient under perfect diffuse illumination. The reflection of the diffuse light that crosses the refractive surface in the other direction, from plastic to air, is called the internal reflection because the reflection occurs within the material medium. In this case the numerical result of the computation is $r_{1.5,1}=0.596$.

Note that for diffuse light, internal reflection values are always much higher than those for surface reflection. When the first medium has a higher refractive index than the second, according to Snell's law there exists a limit incidence angle above which light reflects totally. This total reflection is responsible for the high values of $r_{n_{1}, n_{2}}$ when $n_{1}>n_{2}$.

\section{Simple reflection model}

Assume we print a uniform layer of light-absorbing ink on paper that behaves like an opaque diffuse reflector of reflectance $R_{g}$. The printed ink behaves like a lightabsorbing filter in optical contact with the paper; this means no air layer separates ink and paper. To model this system, we follow the path of the diffuse light flux. The incident flux first crosses the refractive surface between air and ink, then travels through the ink layer where absorption takes place. Since the ink is in optical contact with the paper, the paper reflects the flux like a diffuse reflector. The light flux is oriented upwards and goes through the light-absorbing ink layer a second time. Finally, it crosses the ink-air interface going from the ink layer into the air (see Figure 5).

We must take three different processes into account here: the crossing of the air-ink layer interface, the absorption of the light in the ink, and the reflection on the paper. First we'll consider the flux balance at the airink interface. Only then will we also incorporate the light absorption in the ink and the reflection on the paper. We distinguish here between two diffuse light fluxes: one oriented downwards, denoted $i$, and the other oriented upwards, denoted $j$.

Let's first consider the flux balance at the air-ink interface (see Figure 6). As the incident light flux $i$ hits the interface, a fraction $r_{s} i$ is reflected and the remaining amount $\left(1-r_{s}\right) i$ is transmitted into the ink-layer. Note that the surface reflectance $r_{s}$ equals the average reflection coefficient when light goes from the air into the inklayer of refractive index $n$ : 


$$
r_{s}=\int_{0}^{\frac{\pi}{2}}\left(r_{1, n}(\theta) \cdot \sin 2 \theta\right) d \theta
$$

In the same way, the internal reflection $r_{i}$ equals the average reflection coefficient for diffuse light:

$$
r_{i}=\int_{0}^{\frac{\pi}{2}}\left(r_{1, n}(\theta) \cdot \sin 2 \theta\right) d \theta
$$

The upward-oriented flux inside the ink layer, $j(X)$, hits the interface on the internal side. While the fraction $r_{i} j(X)$ is internally reflected, the remaining $\left(1-r_{i}\right) j(X)$ is transmitted to the air. The balance of the fluxes leads to a set of two linear equations (see Figure 6):

$$
\left\{\begin{array}{c}
i(X)=\left(1-r_{s}\right) i+r_{i} j(X) \\
j=r_{s} i+\left(1-r_{i}\right) j(X)
\end{array}\right.
$$

which we can reorder into a matrix to express $i$ and $j$, the fluxes in air, as a function of $i(X)$ and $j(X)$, the fluxes in the ink layer:

$$
\left[\begin{array}{l}
i \\
j
\end{array}\right]=\left[\begin{array}{cc}
\frac{1}{1-r_{s}} & \frac{-r_{i}}{1-r_{s}} \\
\frac{r_{s}}{1-r_{s}} & \left(1-r_{i}-\frac{r_{i} r_{s}}{1-r_{s}}\right)
\end{array}\right]\left[\begin{array}{c}
i(X) \\
j(X)
\end{array}\right]
$$

We call this matrix the interface correction matrix.

Due to the high value of the internal reflection coefficient $r_{i}$ (see the previous section), more than half of the light flux that has penetrated the ink layer must go several times back and forth between the interface and the paper. These multiple internal reflections cause a nonlinear light absorption. This phenomenon-well known in the literature (see Color Physics for Industry ${ }^{6}$ )—was studied by Saunderson, ${ }^{7}$ who proposed a correcting equation taking it into account. As we'll see, we can derive Saunderson's formula (Equation 27) using the interface correction matrix.

Now, let's look at the second process mentioned above: light absorption in the ink layer. According to the generalization of Beer's law for diffuse light (see Equation 10), the following system of equations determines the variations of the fluxes $i(x)$ and $j(x)$ :

$$
\left\{\begin{array}{l}
\frac{d i(x)}{d x}=K i(x) \\
\frac{d j(x)}{d x}=-K j(x)
\end{array}\right.
$$

where $K=2 \varepsilon(\lambda) \cdot c \cdot \ln (10)$. By abuse of language, $K$ is called the "absorption coefficient" of the ink under diffuse light, whereas it differs from the absorption by a factor $\ln (10)$. Note that due to the axis' upward orien-
5 Simple reflection model for an ink layer of thickness $X$ in optical contact with the paper. The arrows represent diffuse light fluxes. This means that light comes from all directions of one hemisphere with an angular distribution corresponding to the one produced by a Lambert surface.
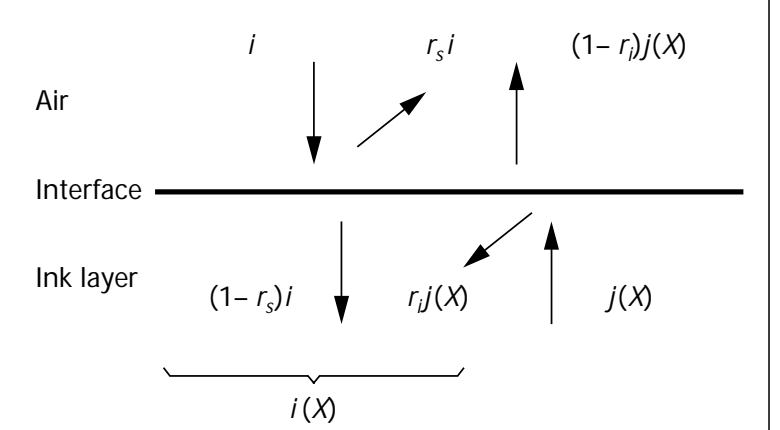

tation, the first equation of the system in Equation 19 contains no minus sign. The boundary condition on the bottom of the ink layer is given by the reflectance $R_{g}$ of the paper: $j(0)=R_{g} \cdot i(0)$, where $i(0)$ and $j(0)$ represent the intensities of the fluxes $i$ and $j$ at $x=0$. For reasons that will appear clearly in the next section, we rewrite Equation 19 in a matrix form:

$$
\frac{d}{d x}\left[\begin{array}{l}
i(x) \\
j(x)
\end{array}\right]=\left[\begin{array}{cc}
K & 0 \\
0 & -K
\end{array}\right] \cdot\left[\begin{array}{l}
i(x) \\
j(x)
\end{array}\right]
$$

This kind of matrix differential equation has a wellknown solution given by the exponential of the matrix. ${ }^{8}$ Note that the exponential of a matrix $\mathbf{M}$ is defined by the power series:

$$
\exp (\mathbf{M})=\sum_{i=0}^{\infty} \frac{\mathbf{M}^{i}}{i !}
$$

Here again, the exponential function plays a central role in the resolution of the differential equation. Since we have to perform the integration of Equation 20 between $x=0$ and $x=X$, the solution is

$$
\begin{aligned}
{\left[\begin{array}{l}
i(X) \\
j(X)
\end{array}\right] } & =\exp \left(\left[\begin{array}{cc}
K & 0 \\
0 & -K
\end{array}\right](X-0)\right) \cdot\left[\begin{array}{c}
i(0) \\
j(0)
\end{array}\right] \\
& =\left[\begin{array}{cc}
\exp (K X) & 0 \\
0 & \exp (-K X)
\end{array}\right] \cdot\left[\begin{array}{c}
i(0) \\
j(0)
\end{array}\right]
\end{aligned}
$$

The ratio $\rho=j(X) / i(X)$ is called the body (or true) reflectance of the analyzed sample. It corresponds to an internal reflection coefficient that doesn't take multiple
6 Flux balance at the air-ink layer interface. 
7 A scattering medium of thickness $X$ in optical contact with a substrate of reflectance $R_{g}$. This medium is divided into parallel layers of infinitesimal thickness $\mathrm{dx}$. We consider two fluxes: $i(x)$ oriented downwards and $\mathrm{j}(\mathrm{x})$ oriented upwards.

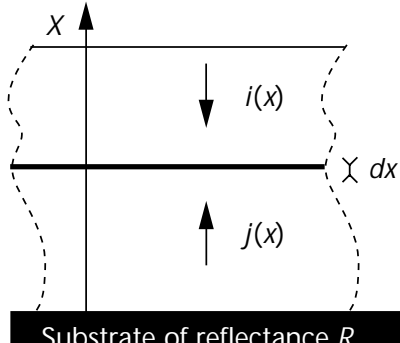

Substrate of reflectance $R_{\mathrm{g}}$

internal reflections into account- the light crosses the ink layer only twice. From Equation 22 and the boundary condition $j(0)=R_{g} \cdot i(0)$, which represents the reflection on the paper (the third process mentioned above), we deduce

$$
\rho=\exp (-2 K X) \cdot R_{g}
$$

However, since real samples are measured in air, we must take into account the changing refractive index, which causes multiple internal reflections. Hence we have to combine Equation 22 with Equation 18 to express the fluxes $i$ and $j$ in air as linear combinations of the fluxes $i(0)$ and $j(0)$. We can write the solution as a matrix product with the boundary condition $j(0)=R_{g} \cdot i(0)$ :

$$
\begin{aligned}
& {\left[\begin{array}{l}
i \\
j
\end{array}\right]=} \\
& {\left[\begin{array}{cc}
\frac{1}{1-r_{s}} & \frac{-r_{i}}{1-r_{s}} \\
\frac{r_{s}}{1-r_{s}} & \left(1-r_{i}-\frac{r_{i} r_{s}}{1-r_{s}}\right)
\end{array}\right] \cdot \exp \left(\left[\begin{array}{cc}
K & 0 \\
0 & -K
\end{array}\right](X-0)\right) \cdot\left[\begin{array}{l}
i(0) \\
j(0)
\end{array}\right]}
\end{aligned}
$$

Let us denote by $t, u, v$, and $w$ the elements of the product matrix:

$$
\left[\begin{array}{l}
i \\
j
\end{array}\right]=\left[\begin{array}{ll}
t & u \\
v & w
\end{array}\right] \cdot\left[\begin{array}{l}
i(0) \\
j(0)
\end{array}\right]
$$

These coefficients combined with the boundary condition $j(0)=R_{g} \cdot i(0)$ enable us to calculate the reflection coefficient $R$ of the analyzed sample. By definition, $R$ corresponds to the ratio of reemitted to incident light. Hence, we have

$$
R=\frac{j}{i}=\frac{t+R_{g} \cdot u}{v+R_{g} \cdot w}
$$

If we develop the product in Equation 26 algebraically, we obtain the well-known Saunderson corrected reflection formula: ${ }^{7}$

$$
R=\frac{j}{i}=r_{s}+\frac{\left(1-r_{s}\right) \cdot\left(1-r_{i}\right) \cdot \rho}{1-r_{i} \rho}
$$

where $\rho$ represents the body reflection of the sample defined in Equation 23.

Thanks to Equation 26, we can predict the reflection

spectrum of a purely light-absorbing ink layer printed on paper. But the most interesting aspect lies in the matrix form of Equation 24. Instead of using several functions incorporated within each other, we simply model the analyzed sample using the product of two matrices. Furthermore, we can easily derive all classical results (body absorption according to Beer's law, Saunderson corrected reflection formula, and so on) from this formulation.

\section{Scattering media}

Plastics and pigmented inks don't just absorb light, they also scatter it. In 1931 Kubelka and Munk ${ }^{9}$ proposed a reflection model based on two diffuse light fluxes in a scattering and absorbing medium, often called the Kubelka-Munk model or the two-flux model in the literature. Let's study a sample made of a scattering and absorbing medium of known thickness $X$ in optical contact with a substrate behaving as a diffuse reflector of reflectance $R_{g}$ (see Figure 7). One flux, denoted $i(x)$, is oriented downwards and the second one, denoted $j(x)$, is oriented upwards.

Let's analyze the variation of these fluxes when they cross a layer of infinitesimal thickness $d x$. Once again, the $x$ axis is oriented upwards, and the origin lies at the top of the substrate. Let $K$ represent the absorption coefficient as defined in the previous section and $S$ the scattering coefficient corresponding to the fraction of the light flux scattered backwards.

We first analyze the variation of $j(x)$ when it crosses the layer. According to Beer's law, this reduces the flux $j(x)$ by an amount $K j(x) d x$, and the back scattering reduces the flux by an amount $S j(x) d x$. The backscattered radiation will increase the flux $i(x)$. In the same way, the flux $j(x)$ receives the light back scattered when $i(x)$ crosses the same layer. Putting these elements together leads to the following equation:

$$
\frac{d j(x)}{d x}=-(K+S) j(x)+\operatorname{Si}(x)
$$

The same analysis performed for the flux $i$ leads to a similar relation (notice the orientation of the axis):

$$
\frac{d i(x)}{d x}=(K+S) i(x)-S j(x)
$$

Together, Equations 28 and 29 provide a system of linear differential equations we can write in matrix form:

$$
\left[\begin{array}{c}
\frac{d i(x)}{d x} \\
\frac{d j(x)}{d x}
\end{array}\right]=\left[\begin{array}{cc}
K+S & -S \\
S & -(K+S)
\end{array}\right] \cdot\left[\begin{array}{c}
i(x) \\
j(x)
\end{array}\right]
$$

We can easily integrate this kind of differential equation between $x=0$ and $x=X$. The solution is given by the exponential ${ }^{8}$ of the matrix in Equation 30: 


$$
\left[\begin{array}{c}
i(X) \\
j(X)
\end{array}\right]=\exp \left(\left[\begin{array}{cc}
K+S & -S \\
S & -(K+S)
\end{array}\right](X-0)\right) \cdot\left[\begin{array}{c}
i(0) \\
j(0)
\end{array}\right]
$$

where $i(0)$ and $j(0)$ represent the fluxes at the top of the substrate, related to each other by the reflectance of the substrate $R_{g}: j(0)=R_{g} \cdot i(0)$. The fluxes $i(X)$ and $j(X)$ correspond respectively to the downward and upward light flux at the top of the scattering layer. We call the matrix in Equation 31 the Kubelka-Munk matrix. Note that for $S=0$ (no scattering), we have the same result as in Equation 22. By developing Equation 31 with the boundary condition $j(0)=R_{g} \cdot i(0)$, we deduce the body reflectance $\rho=j(X) / i(X)$ of the sample. We can derive all remarkable results of the Kubelka-Munk theorylisted in the literature ${ }^{10}$ - from the expression of the body reflectance through algebraic manipulations (see Emmel, ${ }^{11}$ pp. 55-58).

Since the refractive index of the layer differs from that of air, we have to take this change into account by using the interface correction matrix (see Equation 18). The following relation expresses $i$ and $j$ in air as linear combinations of $i(0)$ and $j(0)$ :

$$
\begin{aligned}
{\left[\begin{array}{l}
i \\
j
\end{array}\right]=} & {\left[\begin{array}{cc}
\frac{1}{1-r_{s}} & \frac{-r_{i}}{1-r_{s}} \\
\frac{r_{s}}{1-r_{s}} & \left(1-r_{i}-\frac{r_{s} r_{i}}{1-r_{s}}\right)
\end{array}\right] } \\
& \cdot \exp \left(\left[\begin{array}{cc}
K+S & -S \\
S & -(K+S)
\end{array}\right](X-0)\right) \cdot\left[\begin{array}{l}
i(0) \\
j(0)
\end{array}\right] \\
= & {\left[\begin{array}{ll}
t & u \\
v & w
\end{array}\right] \cdot\left[\begin{array}{l}
i(0) \\
j(0)
\end{array}\right] }
\end{aligned}
$$

We denote the elements of the product matrix by $t, u, v$, and $w$. These coefficients and the boundary condition $j(0)=R_{g} \cdot i(0)$ permit calculating the reflection coefficient $R$ :

$$
R=\frac{j}{i}=\frac{t+R_{g} \cdot u}{v+R_{g} \cdot w}
$$

This final equation allows computing the reflection spectrum of a light-absorbing and light-scattering ink layer printed on a substrate (in our case, paper) of known reflectance $R_{g}$. Once again, the interesting aspect of this study lies in the matrix formulation of Equation 32, which gives a better overview of the modeled system.

This model can also account for the light-scattering phenomenon fluorescence. Note that fluorescence introduces a dependence between different wavelengths, which implies the use of larger matrices, but the general framework remains unchanged. You'll find the corresponding mathematical development in our previous work. ${ }^{12,13}$

\section{Multiple layers}

Some surfaces consist of several superposed layers in optical contact. For example, in color printing the color blue results from superposing a magenta ink layer and a cyan ink layer. The various elements we have seen to this point also let us solve the prediction problem for such materials. We can model each additional layer by the product of two matrices: an interface correction matrix and a Kubelka-Munk matrix.

Consider a color print made of $p$ superposed ink layers. We can model this sample with the product of $2 p$ matrices. Starting from the paper (substrate) where we have the boundary condition $j(0)=R_{g} \cdot i(0)$, we alternately multiply the $(i(0), j(0))$ vector by a Kubelka-Munk matrix $\mathbf{M}_{K}$ and by an interface correction matrix $\mathbf{C}_{K}$ :

$$
\begin{aligned}
{\left[\begin{array}{c}
i \\
j
\end{array}\right] } & =\mathbf{C}_{p} \cdot \mathbf{M}_{p} \cdot \ldots \cdot \mathbf{C}_{k} \cdot \mathbf{M}_{k} \cdot \ldots \cdot \mathbf{C}_{1} \cdot \mathbf{M}_{1} \cdot\left[\begin{array}{l}
i(0) \\
j(0)
\end{array}\right] \\
& =\left[\begin{array}{ll}
t & u \\
v & w
\end{array}\right] \cdot\left[\begin{array}{l}
i(0) \\
j(0)
\end{array}\right]
\end{aligned}
$$

where

$$
\mathbf{M}_{k}=\exp \left(\left[\begin{array}{cc}
K_{k}+S_{k} & -S_{k} \\
S_{k} & -\left(K_{k}+S_{k}\right)
\end{array}\right] X_{k}\right)
$$

and

$$
\mathbf{C}_{k}=\left[\begin{array}{cc}
\frac{1}{1-r_{s, k}} & \frac{-r_{i, k}}{1-r_{s, k}} \\
\frac{r_{s, k}}{1-r_{s, k}} & \left(1-r_{i, k}-\frac{r_{s, k} r_{i, k}}{1-r_{s, k}}\right)
\end{array}\right]
$$

$K_{k}$ and $S_{k}$ respectively represent the absorption and scattering coefficients of layer $k$, and $X_{k}$ the thickness of the layer. Since $\mathbf{C}_{k}$ is an interface correction between the media of layers $k$ and $k+1$ (layer $k+1$ being on top of layer $k$ ), the surface reflection coefficient $r_{s, k}$ and the internal reflection coefficient $r_{i, k}$ are given by

$$
r_{s, k}=\int_{0}^{\frac{\pi}{2}}\left(r_{n_{k+1}, n_{k}}(\theta) \cdot \sin 2 \theta\right) d \theta
$$

and

$$
r_{i, k}=\int_{0}^{\frac{\pi}{2}}\left(r_{n_{k}, n_{k+1}}(\theta) \cdot \sin 2 \theta\right) d \theta
$$

where $n_{k}$ represents the refractive index of layer $k$. Note that for two successive layers with the same refractive index, the interface correction matrix equals the identity matrix.

Finally, we compute the reflection coefficient $R$ of a multilayer color print by using Equation 33 and introducing the resulting values of $t, u, v$, and $w$ computed from Equation 34.

\section{Conclusion}

Our new mathematical framework for color prediction models permits integrating traditional color prediction models (Beer's absorption law, the Fresnel reflection, the Saunderson correction for multiple internal reflections, and the Kubelka-Munk model for scattering media). Consequently, these traditional models no longer exist as separate subjects applying to different situations, but as various particular cases of the same 
unified model. Since the mathematical expressions resulting from our approach contain only the exponential function, they are much simpler than traditional equations-particularly the Kubelka-Munk equations, whose original expressions include hyperbolic functions. The matrix notation also allows clear and easy modeling of multilayer samples.

This new model makes it easy to compute the reflection spectra of printed samples made of uniform ink layers, since the problem reduces to matrix products and exponential functions. The model assumes ideal conditions: perfect diffuse reflector, uniform layers, and planar interfaces. It doesn't take into account particularities such as the violation of Beer's law. But in close to ideal conditions using high-quality paper and soluble dye inks, we've achieved accurate spectral color predictions. Using an extended version of this model, in a previous study ${ }^{13}$ we predicted the spectra of fluorescent samples with an average prediction error of $\Delta E=2.3$ in CIELAB. Furthermore, we computed the spectra of multilayer systems made of superposed Wratten filters on top of a white reflector with high accuracy (see Emmel, ${ }^{11}$ pp. 7980); all elements were in optical contact.

Our model is general and can serve for other applications, including plastics, ceramics, paints, and so forth. Future work will extend this model to halftoned samples, where the halftoning method and light scattering in paper play a major role in the synthesis of the resulting color.

\section{Acknowledgments}

We would like to thank Isaac Amidror for reviewing the paper before submission. We are grateful to the Swiss National Science Foundation, which partly financed this research (grant 21-54127-98).

\section{Bibliography}

1. H.-H. Perkampus, Encyclopedia of Spectroscopy, Wiley-VCH, Weinheim, Germany, 1995, pp. 63-64.

2. G. Wyszecki and W.S. Stiles, Color Science: Concepts and Methods, Quantitative Data and Formulae, 2nd Edition, John Wiley and Sons, New York, 1982.

3. M. Born and E. Wolf, Principles of Optics, Sixth Edition, reissued by Cambridge University Press, Cambridge, UK, 1997.

4. G. Kortüm, Reflectance Spectroscopy, Springer, Berlin, 1969, p. 108.

5. D.B. Judd, "Fresnel Reflection of Diffusely Incident Light," J. National Bureau of Standards, Vol. 29, Nov. 1942, pp. 329-332.

6. Color Physics for Industry, R. McDonald, ed., The Society of Dyers and Colorists, Bradford, UK, 1997, pp. 304-307.

7. J.L. Saunderson, "Calculation of the Color Pigmented Plastics," J. Optical Society of America, Vol. 32, Dec. 1942, pp. 727-736.

8. W.E. Boyce and R.C. DiPrima, Elementary Differential Equations and Boundary Value Problems, Sixth Edition, John Wiley and Sons, New York, 1997, pp. 401-405.

9. P. Kubelka and F. Munk, "Ein Beitrag zur Optik der Farbanstriche," Zeitschrift für technische Physik, Vol. 12, 1931, pp. 593-601 (in German).
10. D.B. Judd and G. Wyszecki, Color in Business, Science and Industry, Third Edition, John Wiley and Sons, New York, 1975, pp. 427-429.

11. P. Emmel, Modèles de Prédiction Couleur Appliqués à l'Impression Jet d'Encre, PhD thesis No. 1857, Ecole Polytechnique Fédérale de Lausanne (EPFL), Lausanne, Switzerland, 1998, http://diwww.epfl.ch/w3lsp/pub/publications/ colour/thesis-emmel.html (in French).

12. P. Emmel and R.D. Hersch, "A 'One Channel' Spectral Color Prediction Model for Transparent Fluorescent Inks on a Transparent Support," Proc. 5th IST/SID Color Imaging Conf., Society for Imaging Science and Technology, Springfield, Va. and Society for Information Display, Santa Ana, Calif., Nov. 17-20, 1997, pp. 70-77.

13. P. Emmel and R.D. Hersch, "Spectral Color Prediction Model for a Transparent Fluorescent Ink on Paper," Proc. 6th IS\&T/SID Color Imaging Conf., Society for Imaging Science and Technology, Springfield, Va. and Society for Information Display, Santa Ana, Calif., Nov. 17-20, 1998, pp. 116-122.

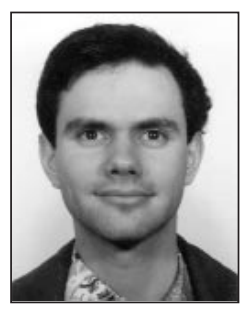

Patrick Emmel is a research assistant at the Peripheral Systems Laboratory of the Swiss Federal Institute of Technology (Ecole Polytechnique Fédérale de Lausanne, or EPFL) in Lausanne, Switzerland. His research interests include color prediction, mathematical modeling of printing processes, spectroscopic measurement of colored inks, color printing with ink-jet methods, and the history of mathematics. He graduated as an electronics and physics engineer from CPE Engineering School in Lyon, France in 1993. The same year he received an MS in computer science from the University Jean Monnet of Saint Etienne, France, and in 1998 he obtained his PhD in computer science from EPFL.

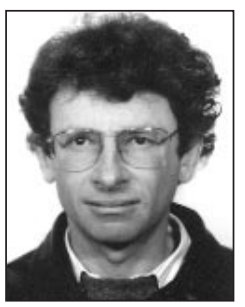

Roger D. Hersch is a professor of computer science and head of the Peripheral Systems Laboratory at the Swiss Federal Institute of Technology (Ecole Polytechnique Fédérale de Lausanne, or EPFL). The lab's main research topics are high-quality color reproduction, digital typography, and parallel image and multimedia storage systems. He received his engineering and PhD degrees from ETH Zurich in 1975 and from EPFL in 1985, respectively. He is the editor of three books and inventor or co-inventor in several patent applications. He served as conference chair for the first International Conference on Raster Imaging and Digital Typography (RIDT 89) and for RIDT 98. He headed the team that developed the Visible Human Slice Server (http://visiblehuman. epfl.ch).

Readers may contact the authors at Laboratoire de Systèmes Périphériques, Ecole Polytechnique Fédérale de Lausanne (EPFL), CH-1015 Lausanne, Switzerland, Patrick.Emmel@epfl.chorRd.Hersch@epfl.ch. 